\title{
Sequential and timely transfection of hepatocyte growth factor and monocyte chemotactic protein-1 ameliorates hyperkinetic pulmonary artery hypertension in rabbits
}

\author{
Yiqian Zhang, MD, ${ }^{\mathrm{a}}$ Fang Zhang, MD, ${ }^{\mathrm{b}}$ Xiaoyu Wang, MD, ${ }^{\mathrm{a}}$ Yue Xie, MD, ${ }^{\mathrm{a}}$ Junjie Du, MD, PhD, \\ Peng Lu, MD, ${ }^{a}$ and Wei Wang, $\mathrm{MD}^{\mathrm{c}}$
}

\begin{abstract}
Objective: To investigate the effect of sequential and timely transfection of the recombinant human hepatocyte growth factor (hHGF) gene and human monocyte chemotactic protein-1 (hMCP-1) gene on hyperkinetic pulmonary artery hypertension in a rabbit model.

Methods: The rabbits with pulmonary artery hypertension were randomly separated into 5 groups: control; hHGF; hMCP-1; hHGF/hMCP-1 simultaneous transfection; and hHGF/hMCP-1 sequential, timely transfection. Two weeks after the transfection, real-time polymerase chain reaction and immunohistochemistry examination were used to detect the expression of hHGF and hMCP-1. Four weeks later, the hemodynamic parameters were measured, and immunohistochemical and immunofluorescence staining were performed, to investigate microvascular density and arterialization.
\end{abstract}

Results: The final adenovirus coding with enhanced green fluorescent protein-hMCP-1 virus was $3 \times 10^{10}$ plaque-forming units $/ \mathrm{mL}$, and the purity of adenovirus coding with hHGF was 1.31. Three days after the transfection, enhance green fluorescent protein hMCP-1 green fluorescence was detected in the lung tissues and increased to its peak point in 1 week. Two weeks later, hHGF and hMCP-1 were expressed in all transfection groups. By the end of 4 weeks, the mean pulmonary artery pressure in the hHGF/hMCP-1 sequential and timely transfection group was lower than that in the other groups. Confirmed by immunohistochemical and immunofluorescence staining, the microvascular and arteriolar density in the lung tissues of the sequential and timely hHGF/hMCP-1 transfection group were higher than that in the other groups.

Conclusions: Expression of hHGF and hMCP-1 were found in rabbit lung after gene transfection via an airway approach. By increasing the pulmonary microvascular density and promoting arterializations, sequential and timely hHGF/hMCP-1 transfection ameliorates the shunt flow-induced pulmonary artery hypertension. (J Thorac Cardiovasc Surg 2015;150:634-43)

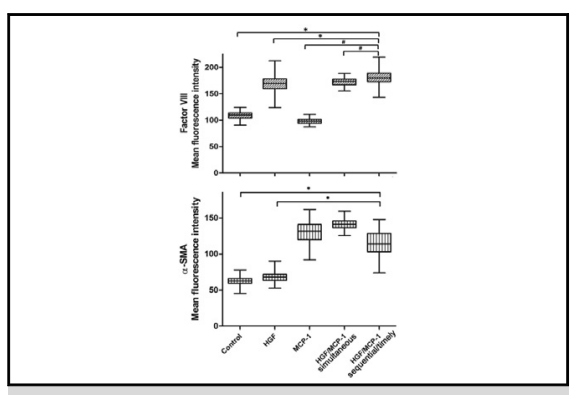

Results of factor VIII (microvascular density) and $\alpha$-SMA (arteriole distribution) staining of lung tissues. * and \#, $P<$ .05, 1-way ANOVA with Fisher-Hayter least significant difference procedure.

\section{Central Message}

Expression of hHGF and hMCP-1 were found in rabbit lungs after gene transfection via an airway approach. By increasing the pulmonary microvascular density and promoting arterializations, sequential and timely hHGF/hMCP-1 transfection ameliorates shunt flowinduced pulmonary artery hypertension.

\section{Perspective}

Hyperkinetic PAH caused by a left-to-right shunt in congenital heart disease is characterized by a decrease of the available pulmonary vascular bed. Development of new therapeutic strategies is critical, to improve the prognosis of this devastating disease. Gene-based angiogenesis therapy is an area of increasing interest to researchers hoping to reduce vascular resistance and improve tissue perfusion. However, the puzzle of how to transform immature neovascular vessels into functional arterioles remains unsolved. We demonstrated that sequential and timely transfection of HGF/MCP-1 ameliorates PAH in rabbits by promoting the growth and arterialization of neovascular microvessels, a new approach to treating PAH.

See Editorial Commentary page 643.
From the Department of a Cardiothoracic Surgery, Affiliated Hospital of Xuzhou Medical College, Xuzhou, Jiangsu; Department of ${ }^{\mathrm{b}}$ Rheumatology, Jiangsu Province Academy of Traditional Chinese Medicine, Nanjing, Jiangsu; and Department of ${ }^{c}$ Cardiothoracic Surgery, First Affiliated Hospital of Nanjing Medical University, Nanjing, People's Republic of China.

This work was supported by the National Natural Science Funding of People's Republic of China (No.30571845), the Natural Science Foundation of Jingsu Province of People's Republic of China (Grant BK2007033), and the Technology Bureau of Xuzhou of People's Republic of China (Grant KC14SH106).
Received for publication Dec 16, 2014; revisions received March 17, 2015; accepted for publication March 29, 2015; available ahead of print May 1, 2015.

Address for reprints: Wei Wang, MD, Department of Cardiothoracic Surgery, First Affiliated Hospital of Nanjing Medical University, 300 Guangzhou Rd, Jiangsu Province, Nanjing 210029, People's Republic of China (E-mail: 18052268137@ 163.com). $0022-5223 / \$ 36.00$ Copyright () 2015 by The American Association for Thoracic Surgery http://dx.doi.org/10.1016/j.jtcvs.2015.03.046 


\section{Abbreviations and Acronyms \\ Ad = adenovirus \\ EGFP $=$ enhanced green fluorescent protein \\ hHGF = human hepatocyte growth factor \\ HGF = hepatocyte growth factor \\ hMCP-1 = human monocyte chemotactic protein-1 \\ MCP-1 = monocyte chemotactic protein-1 \\ mRNA $=$ messenger ribonucleic acid \\ PAH = pulmonary arterial hypertension \\ $\mathrm{Pfu} \quad=$ plaque-forming units \\ SMA $=$ smooth muscle actin \\ SMC $=$ smooth muscle cell \\ VEC $=$ vascular endothelial cell}

Hyperkinetic pulmonary artery hypertension (PAH) is a common and problematic complication of congenital heart disease with left-to-right shunt. This condition is associated with increased mortality and therefore seriously affects the prognosis of such patients. ${ }^{1}$ Previous studies suggested that acinar pulmonary arteries are key regulators of pulmonary hemodynamics and luminal stenosis, owing to the fact that thickening pulmonary arterial walls contribute to $\mathrm{PAH}^{2}$

Currently, vascular dilatation, via inhalation of nitrous oxide, with use of nitric acid lipid drugs and prostacyclin, is recommended as a first approach to treating the disease. ${ }^{3,4}$ Although palliation of PAH can be achieved with surgical manipulation, ${ }^{5,6}$ and pharmacotherapies can prolong the survival of patients with $\mathrm{PAH}^{7}$ the long-term outcome is not favorable because of the obstructive remodeling of pulmonary arterioles and the decrease in the available pulmonary vascular bed. ${ }^{8}$ In recent years, gene-based angiogenesis therapy has become an area of increasing interest in many research fields. Therapeutic angiogenesis reduces vascular resistance and improves perfusion of tissues. ${ }^{9,10}$

Hepatocyte growth factor (HGF) is a multifunctional growth factor evolving from mesenchymal origin, bound to its specific membrane receptor, c-met, to induce diverse physiologic activities. ${ }^{11}$ In recent years, several studies have shown that HGF can promote endothelial cell mitosis more effectively than vascular endothelial growth factor, without increasing vascular permeability. ${ }^{12,13}$ Furthermore, it can suppress vascular endothelial cell (VEC) apoptosis, and protect neovascularization from degradation. ${ }^{11}$ Our previous study suggested that instilling Ad-hHGF could effectively transfect alveolar epithelium, bronchial epithelium, and pulmonary VECs, and allow the microvessels in the lung tissues to fluorish. But most of them were only nonmuscular, immature microvasculature, limiting their function to reducing pulmonary artery pressure. ${ }^{14}$

The method of transforming neovascular vessels into arterioles is critical to effective reduction of pulmonary artery pressure. Monocyte chemotactic protein-1 (MCP-1), usually secreted by VECs, smooth muscle cells (SMCs), or macrophages, is a chemotactic factor belonging to the chemotactic cytokine family. It can promote the mitosis of vascular endothelium, adhere to CCR2 receptor, and evoke oriented chemotaxis of VECs. ${ }^{15}$ Furthermore, MCP-1 can promote the mitosis of VECs, and urge VECs and mesenchymal cells to move toward VECs, thereby accelerating the process of neovascular arterialization. ${ }^{16}$

In this study, hHGF was transfected in the lungs of PAH rabbit models to induce the development of pulmonary microvessels. Afterward, hMCP-1 was sequentially transfected in a timely fashion to promote the growth and arterialization of neovascular microvessels. The collateral circulation with physiologic function was constructed to decrease the impedance of the pulmonary bloodstream and the pulmonary blood pressure. The experimental study was designed to determine whether sequentially and timely transfected HGF/MCP-1 can be used in treating pulmonary hypertension, and its effectiveness.

\section{METHODS}

\section{Animal Models}

The study was conducted in accordance with the Guide for the Care and Use of Laboratory Animals of the National Research Council of China. The experiment was approved by the Animal Care Committee of the Xuzhou Medical College of China. The animals received humane care in compliance with the Guide for the Care and Use of Laboratory Animals of Jiangsu Province of China. One-month-old New Zealand male rabbits (weighing $540 \pm 30 \mathrm{~g}$ ) were supplied by the Laboratory Animal Center of Xuzhou Medical College. A systemic-pulmonary shunt was established with left common carotid artery and pulmonary trunk anastomosis, as described previously. ${ }^{17}$ The pulmonary hypertension model was established after 3 months. A total of 110 rabbits were utilized, and 77 rabbits survived after 3 months, a survival rate of $70 \%$. In addition, 12 healthy rabbits were used for detecting hMCP-1 expression in lung tissue.

\section{Detection of hMCP-1 Expression}

Twelve normal rabbits were randomly separated into 4 groups, and endotracheally instilled with Ad-enhanced green fluorescent protein (EGFP)-hMCP-1 $\left(2 \times 10^{9}\right.$ Pfu per rabbit). Enhanced green fluorescent protein-hMCP-1 fluorescence and real-time polymerase chain reaction were employed to detect expression of hMCP-1 in the lung tissues.

\section{Preparation of Recombinant Adenoviral Vector}

Recombinant adenovirus (replication-defective human serum adenovirus type 5 with cytomegalovirus promoter) coding with human HGF (Ad-hHGF) were constructed by the Academy of Military Medical 
Sciences and donated by Dr Wu Zuze. The efficacy and safety of Ad-hHGF has been demonstrated by Ha and colleagues. ${ }^{18}$ The purity of Ad-hHGF and titer was 1.32 and $2.3 \times 10^{10} \mathrm{pfu} / \mathrm{mL}$, respectively. ${ }^{18}$

In our own facility, we constructed an Ad-EGFP-hMCP-1 recombinant adenovirus vector. The original plasmid (pINCY) carrying the hMCP-1 gene was purchased from Open Biosystems Corporation of America (Lafayette, Colo). The vector plasmid (pDC315-EGFP Vector) and E. coli strains DH5 $\alpha$ used for the amplification of adenovirus were from Genkey Corporation (Shanghai, People's Republic of China). The auxiliary packaging plasmid (pBGH lox $\Delta \mathrm{e} 1,3 \mathrm{cre}$ ) was purchased from Microbix Biosystems, Inc (Mississauga, Canada). The recombinant Ad vector with 2 helper plasmids was cotransfected in HEK293T cells by the calcium phosphate precipitation method. The design of the polymerase chain reaction primers was carried out according to hMCP-1 mRNA (messenger ribonucleic acid) indexed in GeneBank (NM_002982): (1) hMCP-1-EcoRIF: GTAGAACGCAGATCGAATTCATGAAAGTCTCTGCCGCC; (2) MCP-1-EcoRI-R: CCCTTGCTCACCATGAATTCAGTCTTCGGAGTTT GGGTT (Exchange pairing bases (Underline), EcoRI restriction sites (bold), the target gene $3^{\prime}$ end partial sequence (Italic)); (3) EGFP-N-R: CGTCGCCGTCCAGCTCGACCAG, used for identification and sequencing. The primers were synthesized by Invitrogen Corporation (Carlsbad, Calif). The specific band at $337 \mathrm{bp}$ represented the polymerase chain reaction product of hMCP-1 (Figure E1). The final Pfu of virus were determined by titration on HEK293T cells under an agar overlay (Sigma-Aldrich, St Louis, Mo). The virus was stored at $-80^{\circ} \mathrm{C}$ in the dark, until it was used again.

\section{Experimental Protocol}

The rabbits with established PAH $(\mathrm{n}=75)$ were randomly separated into 5 groups (15 per group). All treatments were administered via intratracheal instillation, at a dosage of $2 \times 10^{9} \mathrm{Pfu}$. Group A (control) received Ad containing no gene in the expression cassette (Ad-Null), repeated after 1 week. Group B (Ad-hHGF treatment) received Ad-hHGF, and 1 week later, Ad-Null. Group C (Ad-hMCP-1 treatment) received Ad-hMCP-1, and 1 week later, Ad-Null. Group D (hHGF/hMCP-1 simultaneous treatment) received both Ad-hHGF and Ad-hMCP-1 at the same time. Group E (hHGF/hMCP-1 sequential and timely treatment) received Ad-hHGF, and 1 week later, Ad-hMCP-1 (Table E1). Briefly, after the sterilization of the skin, the trachea was approached via a thyrocricoid puncture, and $200 \mu \mathrm{l}$ of vehicle containing Ad-hHGF, equivalent Ad-hMCP-1, or Ad-Null was instilled into the trachea. Immediately before the instillation, circumferential compression of the thorax was performed to achieve a forced exhalation. After endotracheal instillation, followed by $20 \mathrm{~mL}$ of air, compression was released.

\section{Measurement of Hemodynamic Parameters}

Four weeks after the transfection, animals were anesthetized with an intramuscular injection of ketamine $(2 \mathrm{mg} / \mathrm{kg})$ and midazolam $(1 \mathrm{mg} / \mathrm{kg})$, followed by an intravenous bolus of ketamine $(1 \mathrm{mg} / \mathrm{kg})$ through the ear vein. The animals were intubated and supported with a ventilator. A right thoracotomy was performed through the fourth intercostal space. ${ }^{19} \mathrm{~A}$ purse-string suture was made on the ascending aorta and the pulmonary trunk with 7-0 Prolene (polypropylene, Ethicon Inc, Somerville, NJ). A piezometric catheter $(4 \mathrm{~F})$ was inserted, and the hemodynamic parameters were monitored simultaneously with the MacLab/8s multifunction physiologic instrument (M8680, AD Instruments Pty Ltd, Bella Vista, Australia). Hemodynamic parameters were recorded, including systolic pulmonary artery pressure, diastolic pulmonary artery pressure, mean pulmonary artery pressure, and mean systemic arterial pressure.

\section{Preparation of the Tissues}

Two weeks after transfection, 5 rabbits were taken from each group. Lung samples $(1 \times 1 \times 1 \mathrm{~cm})$ were cut from the apex of the superior lobe of the right lung for real-time polymerase chain reaction. The left lung inferior lobe was fixed by $4 \%$ paraformaldehyde and routinely processed into paraffin sections, $5 \mu \mathrm{m}$ in thickness, for immunostaining examination. Four weeks after transfection, the rest of the animals (10 from each group) were killed with an overdose of $3 \%$ pentobarbital $(90-120 \mathrm{mg} / \mathrm{kg})$ via an intravenous infusion after their hemodynamic parameters had been measured. Lung samples $(1 \times 1 \times 1 \mathrm{~cm})$ were fixed by $4 \%$ paraformaldehyde and routinely processed into paraffin sections, $5 \mu \mathrm{m}$ in thickness, for immunohistochemical analysis and immunofluorescence staining. At the same time, the great vessels were separated from the heart, and the residual blood was blotted. The heart was separated into the right ventricle and left ventricle plus ventricular septum, according to the methods previously described. ${ }^{20}$

\section{Real-Time Polymerase Chain Reaction}

Total RNA (ribonucleic acid) was extracted from the lung tissues with TRIzol Plus RNA Purification Kit (Invitrogen Corporation, Carlsbad, Calif), according to the manufacturer instructions. First, strand cDNA (complementary DNA) was synthesized with random hexanucleotides from $1 \mathrm{mg}$ of total RNA, using GoTaq 2-Step RT-qPCR System (Promega UK Ltd, Southampton, United Kingdom). Quantitative real-time polymerase chain reaction for MCP-1 was performed using SYBR green reagent (Sigma-Aldrich, St Louis, Mo), under the following conditions: $95^{\circ} \mathrm{C}$ for 30 seconds; 30 cycles of $95^{\circ} \mathrm{C}$ for 30 seconds; $55^{\circ} \mathrm{C}$ for 30 seconds; and $72^{\circ} \mathrm{C}$ for 30 seconds; and the final extension at $72^{\circ} \mathrm{C}$ for 10 minutes; and finally, stored at $4^{\circ} \mathrm{C}$. The primers were treated with the following sequences: for the 337-base-long product for hMCP-1: sense, 5'GTAGAACGCAGATCGAATTCATGAAAGTCTCT GCCGCC $3^{\prime}$, and antisense, 5'CCCTTGCTCACCATGAATTCAGTCTT CGGAGTTTGGGTT3 ${ }^{\prime}$; for the 489-base-long product for hHGF: sense, 5'AACAACTGAATGCATCCAAGGTC $3^{\prime}$, and antisense, 5'GCAATAAT CCCAAGGAATGAGTG3'. In addition, the levels of $\beta$-actin mRNA were tested using human $\beta$-actin-primers: sense, $5^{\prime}$ GGCATCGTGATG GACTCCG3', and antisense, 5'GCTGGAAGGTGGACAGCGA3'. The size of the $\beta$-actin product was $407 \mathrm{bp}$. The polymerase chain reaction product band intensities were analyzed using Quantiscan software.

\section{Immunohistochemical Analysis}

Twenty slices were randomly selected in each group to detect the target proteins. The slices were blocked with donkey serum and incubated at $4^{\circ} \mathrm{C}$ overnight with anti-hHGF mouse antibody $(1: 100$, Cruz Biotechnology Inc, Santa Cruz, Calif); anti-hMCP-1 mouse antibody (1:100, Cruz Biotechnology Inc, Santa Cruz, Calif); anti-rat factor VIII rabbit antibody (1:150, Sigma, St. Louis, Mo), anti-rat $\alpha$-SMA (simultaneous multiantibody) goat antibody (1:150, Sigma), or without any antibodies as a control. Biotinylated second antibody was added and kept for 30 minutes at $37^{\circ} \mathrm{C}$. The slides were incubated with avidin-biotin complex for 30 minutes at $37^{\circ} \mathrm{C}$. A DAB kit (Cambridge, United Kingdom) was used for chromogenic reaction, and the slices were counterstained with hematoxylin. Factor VIII and $\alpha$-SMA staining were used to display VECs and SMCs in the lung vessels, as previously described. ${ }^{21}$ The pathologist was blinded to the experiments.

\section{Factor VIII and $\alpha$-SMA Immunofluorescence Double Staining}

Paraffin sections of the lung tissues were treated with hydrogen peroxide and blocked with donkey serum. After an incubation with mixed primary antibody, a mixture of anti-rat factor VIII rabbit antibody (1:150; Sigma) and anti-rat $\alpha$-SMA goat antibody (1:150; Sigma, America), fluorescein isothiocyanate-labeled donkey anti-rabbit secondary antibody and rhodamine-labeled donkey antigoat secondary antibody were used to display VECs and SMCs in the lung vessels, respectively. All 

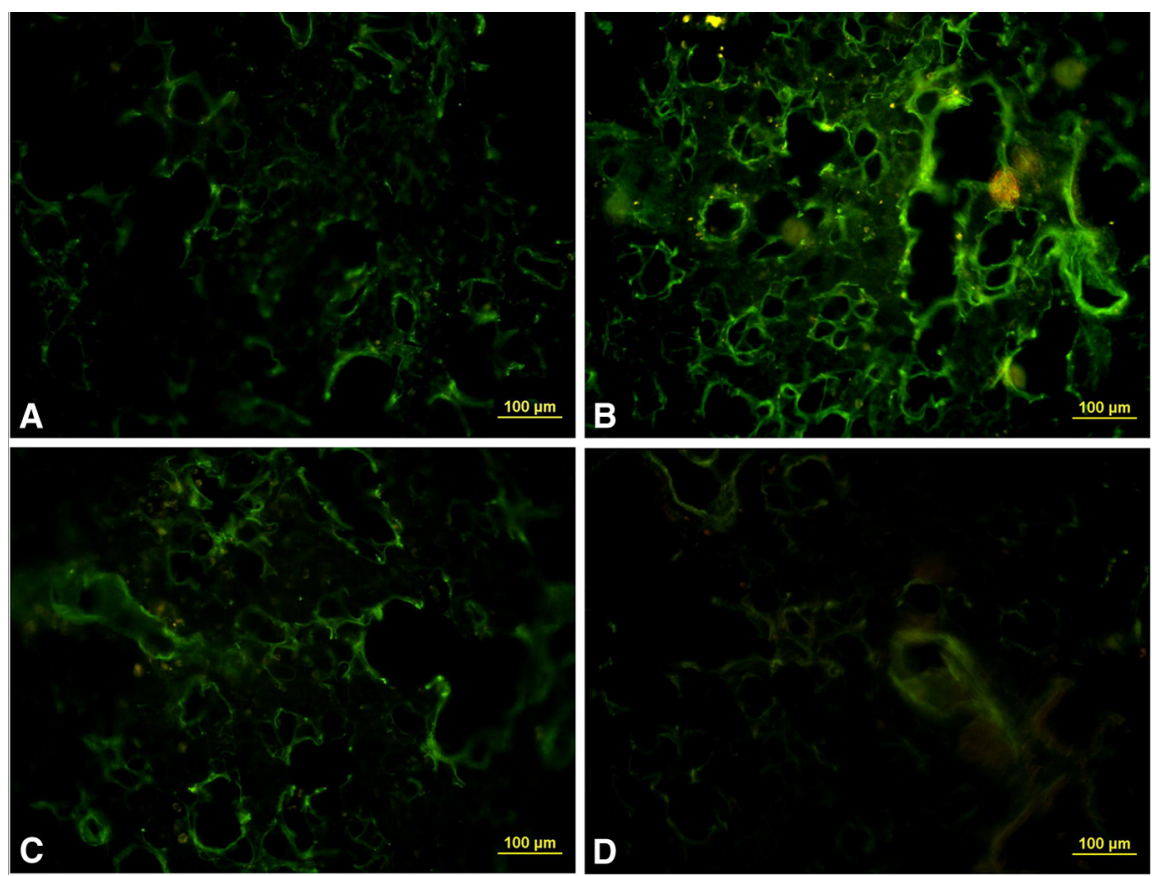

FIGURE 1. The figure shows hMCP-1-EGFP fluorescence expression after transfection at different time points. A, Three days after transfection, green fluorescence was detected in the lung tissues. B, One week later, green fluorescence increased to its peak point. C, Two weeks later, the green fluorescence dissipated. D, Four weeks later, the green fluorescence disappeared. Bar $=100 \mu \mathrm{m}$.

manipulations were performed in the dark. The positive signals and the intensity of the fluorescence were analyzed by the Image Pro Plus 6.0 system.

\section{Statistical Analysis}

Data are shown as mean \pm SD. All statistical analyses were conducted with SPSS, version 13.0 (SPSS Inc, Chicago, Ill) for Windows. One-way ANOVA was performed, with a post-hoc test using the Fisher-Hayter least significant difference procedure.

\section{RESULTS}

\section{Determination of hMCP-1 Expression in Lung Tissue}

The final Ad-EGFP-MCP-1 virus was $3 \times 10^{10} \mathrm{pfu} / \mathrm{mL}$, determined by titration on HEK293T cells under an agar overlay. The purity of Ad-EGFP-MCP-1 was 1.31 . Three days after transfection of Ad-EGFP-MCP-1, EGFP-hMCP-1 green fluorescence was detected in the lung tissues. One week after transfection, the green fluorescence increased to its peak point. Two weeks later, EGFP-hMCP1 green fluorescence began to dissipate. Four weeks later, the green fluorescence disappeared (Figure 1).

\section{Hemodynamic Changes}

No animals died or experienced respiratory or other complications after the gene transfection. Four weeks later, the mean pulmonary artery pressure $(17.96 \pm 1.04 \mathrm{~mm} \mathrm{Hg})$ and the weight index of the right ventricle $(0.37 \pm 0.02 \mathrm{~g} / \mathrm{g})$ in group $\mathrm{E}$ was significantly lower than those in the other groups. The mean pulmonary artery pressure $(21.07 \pm 0.92 \mathrm{~mm} \mathrm{Hg})$ and the weight index of the right ventricle $(0.39 \pm 0.03 \mathrm{~g} / \mathrm{g})$ in group $\mathrm{B}$ was lower than those in groups A $(30.06 \pm 1.44 \mathrm{~mm} \mathrm{Hg}, 0.41 \pm 0.03$ $\mathrm{g} / \mathrm{g}), \mathrm{C}(29.34 \pm 0.86 \mathrm{~mm} \mathrm{Hg}, 0.40 \pm 0.02 \mathrm{~g} / \mathrm{g})$, and D $(29.68 \pm 1.46 \mathrm{~mm} \mathrm{Hg}, 0.39 \pm 0.03 \mathrm{~g} / \mathrm{g})$. No significant differences were found in mean systemic arterial pressure and the weight index of the right ventricle among the 5 groups. After the gene transfection, no difference was noticed in weight or heart rate among the groups (Table 1).

\section{Expression of HGF and MCP-1 mRNA and Protein in Lung Tissue}

Two weeks after the transfection, hHGF mRNA was detected in the lung tissues in groups $\mathrm{B}, \mathrm{D}$, and $\mathrm{E}$, and no hHGF-positive signals were seen in groups $\mathrm{A}$ or $\mathrm{C}$; hMCP-1 mRNA was detected in groups $\mathrm{C}, \mathrm{D}$, and $\mathrm{E}$, and no hMCP-1-positive signals were seen in groups $\mathrm{A}$ and $\mathrm{B} ; \beta$-actin mRNA was detected in each group (Figure 2, A). Immunostaining for hHGF and hMCP-1 was observed in the cytoplasm of VECs, alveolar epithelial cells, and bronchial epithelial cells in groups D and E. In group B, an hHGF-positive signal was observed, but no hMCP-1 signal. In group $\mathrm{C}$, an hMCP-1-positive signal was observed, but no hHGF signal. In group A, no hHGF- or hMCP-1-positive signal was observed (Figure 2, B).

\section{Number of VECs and SMCs}

Factor VIII immunostaining showed that the number of VECs in group E $\left(17.3 \pm 2.0 / \mathrm{mm}^{2}\right)$ was significantly 
TABLE 1. Hemodynamic measurement and weight index of right ventricle

\begin{tabular}{lccccc}
\hline \multicolumn{1}{c}{ Measure } & Group A $(\mathbf{n}=\mathbf{1 0})$ & Group B $(\mathbf{n}=\mathbf{1 0})$ & Group C $(\mathbf{n}=\mathbf{1 0})$ & Group D $(\mathbf{n}=\mathbf{1 0})$ & Group E $(\mathbf{n}=\mathbf{1 0})$ \\
\hline sPAP $(\mathrm{mm} \mathrm{Hg})$ & $41.41 \pm 2.16$ & $33.34 \pm 2.29 \dagger$ & $39.88 \pm 1.92$ & $40.57 \pm 2.39$ & $29.39 \pm 1.94^{*}$ \\
dPAP (mm Hg) & $24.39 \pm 1.30$ & $14.94 \pm 1.18 \dagger$ & $24.07 \pm 1.23$ & $24.23 \pm 1.36$ & $12.24 \pm 1.17^{*}$ \\
mPAP (mm Hg) & $30.06 \pm 1.44$ & $21.07 \pm 0.92 \dagger$ & $29.34 \pm 0.86$ & $29.68 \pm 1.46$ & $17.96 \pm 1.04^{*}$ \\
mSAP (mm Hg) & $75.23 \pm 12.45$ & $79.34 \pm 14.50$ & $72.09 \pm 13.32$ & $78.74 \pm 16.19$ & $71.63 \pm 15.78$ \\
RV/BW (g/kg) & $0.81 \pm 0.35$ & $0.54 \pm 0.23 \dagger$ & $0.79 \pm 0.29$ & $0.76 \pm 0.32$ & $0.46 \pm 0.18^{*}$ \\
LV/BW (g/kg) & $2.46 \pm 0.47$ & $2.65 \pm 0.79$ & $2.98 \pm 0.83$ & $2.83 \pm 0.64$ & $2.58 \pm 0.65$ \\
RV/LV + VS (g/g) & $0.41 \pm 0.03$ & $0.39 \pm 0.03$ & $0.40 \pm 0.02$ & $0.39 \pm 0.03$ & $0.37 \pm 0.02^{*}$ \\
Heart rate (bpm) & $270 \pm 51$ & $254 \pm 39$ & $258 \pm 40$ & $257 \pm 49$ & $263 \pm 47$ \\
\hline Van
\end{tabular}

Values are mean \pm SD. $s P A P$, Systolic pulmonary artery pressure; $d P A P$, diastolic pulmonary artery pressure; $m P A P$, mean pulmonary artery pressure; $m S A P$, mean systemic arterial pressure; $R V$, right ventricle; $B W$, body weight; $L V$, left ventricle; $V S$, ventricular septum. $* P<.05$ versus the other groups. $\dagger P<.05$ versus the $\mathrm{A}$, $\mathrm{C}$, and $\mathrm{D}$ groups.

larger than those in groups A $\left(9.0 \pm 1.7 / \mathrm{mm}^{2}\right)$ and $\mathrm{C}$ $\left(9.2 \pm 1.0 / \mathrm{mm}^{2}\right)$, but noticeably smaller than that in group B $\left(19.2 \pm 2.0 / \mathrm{mm}^{2}\right)$; the number of VECs in group D $\left(15.4 \pm 1.5 / \mathrm{mm}^{2}\right)$ was markedly smaller than that in group $\mathrm{E}$, but remarkably larger than those in groups $\mathrm{A}$ and $\mathrm{C}$. The $\alpha$-SMA immunostaining showed that the number of SMCs in group E $\left(5.5+0.6 / \mathrm{mm}^{2}\right)$ was significantly larger than those in groups A $\left(4.6 \pm 0.3 / \mathrm{mm}^{2}\right)$ and B $\left(2.9 \pm 0.5 / \mathrm{mm}^{2}\right)$, but remarkably smaller than that in groups $\mathrm{C}\left(7.3 \pm 0.6 / \mathrm{mm}^{2}\right)$ and $\mathrm{D}\left(7.52+0.6 / \mathrm{mm}^{2}\right)$ (Figure 3). All results in the 5 groups were compared by 1-way ANOVA with the Fisher-Hayter least significant difference procedure (ANOVA of VECs: $\mathrm{F}=158.52, P<.01$; ANOVA of SMCs: $\mathrm{F}=277.31, P<.01)$. Detailed statistical results are given in Table E2.

\section{Microvascular Density and Microvascular Arterialization}

The mean fluorescence intensity of factor VIII was used to indicate the pulmonary microvascular density: groups $\mathrm{B}$ (169.3 \pm 15.0$), \mathrm{D}(172.7 \pm 7.5)$, and $\mathrm{E}(180.3 \pm 13.3)$ had significantly higher intensity than groups A $(109.3 \pm 6.7)$ and $C(98.0 \pm 5.1)$. The mean fluorescence intensity of $\alpha$-SMA was used to indicate the pulmonary microvascular arterioles: groups C (131.1 \pm 13.6$), \mathrm{D}(141.3 \pm 6.7)$, and $\mathrm{E}(115.0 \pm 16.7)$ had markedly higher intensity than groups A $(62.3 \pm 6.1)$ and $B(67.7 \pm 6.4)$, but group $E$ was noticeably lower than groups $\mathrm{C}$ and D (Figure 4). The results from factor VIII and $\alpha$-SMA immunohistochemical staining were similar to the results given here.

All the results suggested that hHGF induced the proliferation of VECs, and increased microvascular density; hMCP-1 induced proliferation of SMCs, promoted microvascular arterialization, and thickened the microvascular wall. But simultaneously, transfection of hHGF/hMCP-1 led to excessive proliferation of SMCs, microvascular wall thickening, and luminal stenosis; sequential and timely transfection of hHGF/hMCP-1 increased microvascular density and promoted microvascular arterialization. Furthermore, the pulmonary microvascular structure was better than that of $\mathrm{hHGF} / \mathrm{hMCP}-1$ transfected simultaneously. The results were compared by 1-way ANOVA, with the Fisher-Hayter least significant difference procedure (ANOVA of factor VIII: $\mathrm{F}=1430.93, P<.01 ;$ ANOVA of $\alpha$-SMA: $\mathrm{F}=1124.37$, $P<.01)$. Detailed statistical results are given in Table E3.

\section{DISCUSSION}

The current study demonstrated that: (1) The intratracheal transfection of hHGF and hMCP-1, with an interval of 1 week, can induce the expression of hHGF and hMCP-1 in the lung tissues of rabbits with PAH; (2) Sequential and timely expression of hHGF and hMCP-1 can increase microvascular density, promote microvascular arterialization, and generate pulmonary microvessels with better structure; and (3) The sequential and timely expression of hHGF and hMCP-1 can ameliorate the $\mathrm{PAH}$, and may be a result of the neovascularization induced by hHGF, and the ensuing arterialization triggered by hMCP-1.

Hepatocyte growth factor, together with endothelial surface receptors, stimulates endothelial cells and lets them migrate, proliferate, mature, and secret protease. ${ }^{22}$ These endothelial cells, once having moved into the exocellular interstitium, begin to germinate, until capillary-like micovessels appear. ${ }^{23}$ In the lung tissues, HGF is, in addition, a powerful factor in evoking angiogenesis. ${ }^{24}$

In a previous experiment using HvJ liposome, hHGF was transfected in the left lung, through the pulmonary artery. Seven days after the transfection, microvascular density in the left lung was much higher, and the right ventricular pressure was lower than that in the control group; 14 days after, Doppler imaging revealed that the average blood perfusion rate in the left lung was higher than that in the right lung. ${ }^{25-27}$

Preliminary work has shown that singly transfected HGF can generate plenty of capillary-like nonmuscular vessels in lung tissues, and to some extent, lower the pulmonary blood pressure by enlarging the sectional area of the pulmonary blood vessel bed. ${ }^{28}$ However, this effect is not desirable. In this experiment, in group B, transfected with HGF-Ad, 

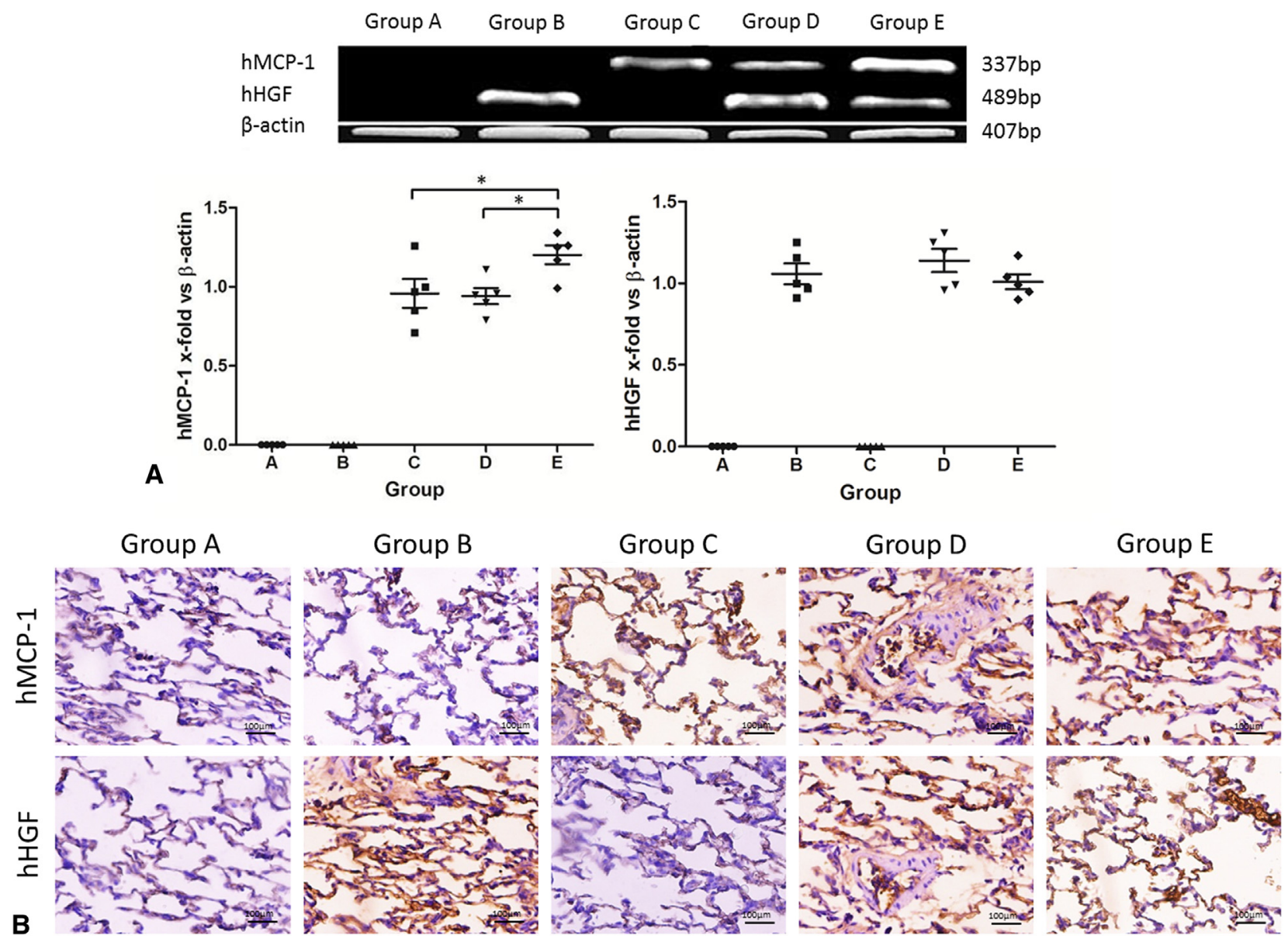

FIGURE 2. The figure shows hMCP-1 and hHGF expression in the lung tissues 2 weeks after transfection. A, Real-time polymerase chain reaction showed that expression of hMCP-1 in group E was higher than that in groups $\mathrm{C}$ and $\mathrm{D}(\mathrm{n}=5, * P<.05)$. No MCP-1-positive signals were seen in groups A or $\mathrm{B}$. Expression of hHGF was detected in groups B, D, and E in the lungs; no hHGF-positive signals were seen in the A and C groups. Symbols depict actual data points; the middle horizontal bar represents mean value; upper and lower bars represent standard error of the mean. B, Immunostaining for hMCP-1 and hHGF was observed in the cytoplasm of vascular endothelial cells, alveolar epithelial cells, and bronchial epithelial cells in groups D and E. An hHGF positive signal, but no hMCP-1-positive signal was observed in group B. An hMCP-1-positive signal, but no hHGF-positive signal was observed in group C. No hHGF or hMCP-1 positive signal was observed in group A. Bar $=100 \mu \mathrm{m}$. $h M C P-1$, Human monocyte chemotactic protein-1 gene; $h H G F$, human hepatocyte growth factor.

the density of endothelial cells and arterioles was significantly higher than that in group A, but the density of the VECs and muscular vessels is almost the same in the 2 groups. These findings demonstrate that the singly transfected HGF is not enough to establish a collateral circulation functioning as the artery.

Monocyte chemotactic protein-1, which is 1 chemotactic factor of the $\mathrm{CC}$ family that was discovered first and widely studied, can be secreted by endothelial cells and macrophages. People are interested in MCP-1 for its potential chemical induction and ability to activate macrophages. ${ }^{29}$ Recent studies suggest that MCP-1 is involved in angiogenesis. However, controversy continues regarding how MCP-1 affects blood vessel parts, such as endothelial cells, vascular VECs, and the basement membrane. $^{30}$
Nevertheless, its effect on proliferation and chemotaxis of vascular VECs has been clarified. The vascular VEC is unique in being the only cell found in the medial arteriole. Whether smooth muscle can develop in newborn vessels determines the endpoint of arterialization.

In this study, the numbers of VECs in groups $\mathrm{C}, \mathrm{D}$, and $\mathrm{E}$ are significantly larger than those in groups $\mathrm{A}$ and $\mathrm{B}$. But in group $\mathrm{C}$, no endothelial proliferation and no effect of MCP-1 on promoting endothelial proliferation are seen; no difference of vascular density is examined, compared with group $\mathrm{A}$. In group D, the number of endothelial cells is slightly smaller than that in group B; its vascular density almost equals that of group C. Plenty of arterioles develop; the smooth muscle layer thickens and migrates into the endothelium, causing vascular luminal stenosis. This change provides evidence that overexpression of MCP-1, 

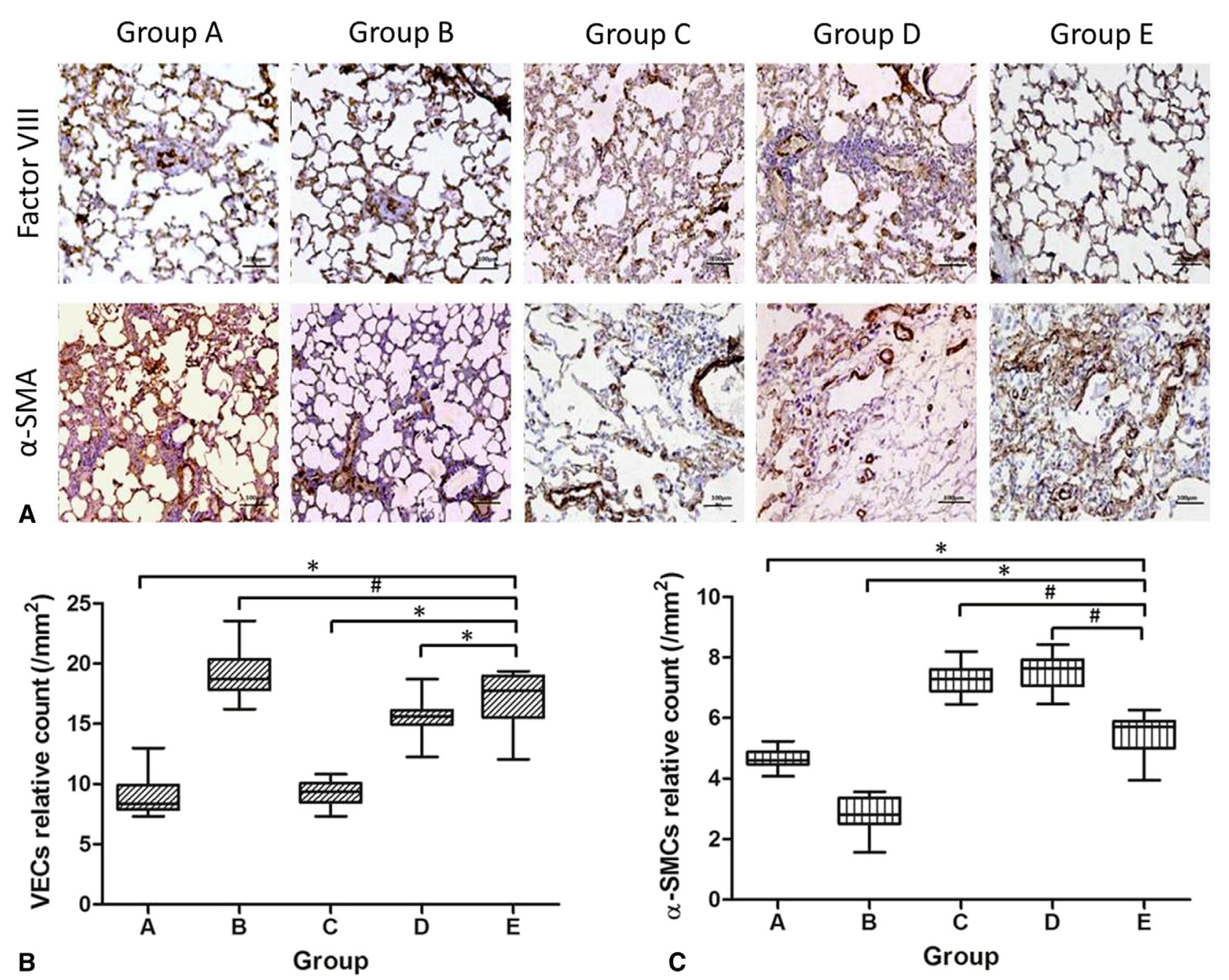

FIGURE 3. Number of VECs and SMCs ( $\mathrm{n}=20$ slices per group). A, Factor VIII and $\alpha$-SMA immunostaining was employed to evaluate the number of VECs and SMCs, respectively, in the lungs after transfection. B, Semiquantitative analysis of the hHGF immunohistochemistry examination. The number of VECs in group $\mathrm{E}$ was greater than that in groups $\mathrm{A}$ and $\mathrm{C}(* P<.05)$, but noticeably smaller than that in group $\mathrm{B}(\# P<.05)$. The number of VECs in group $\mathrm{D}$ was smaller than that in group $\mathrm{E}(* P<.05)$, but remarkably larger than that in groups A and $\mathrm{C}(* P<.05)$. C, Semiquantitative analysis of the hMCP-1 immunohistochemistry examination: The number of SMCs in group E was significantly larger than that in groups A and $\mathrm{B}\left({ }^{*} P<.05\right)$, but remarkably smaller than that in groups $\mathrm{C}$ and $\mathrm{D}(\# P<.05)$. Bar $=100 \mu \mathrm{m}$. B and C, Horizontal bars within the boxes indicate median; the top and bottom of the boxes indicate the 75th and 25th percentiles, respectively (ANOVA of VECs: $\mathrm{F}=158.52, P<.01$; ANOVA of SMCs: $\mathrm{F}=277.31, P<.01 ; *$ and \#, $P<.05,1$-way ANOVA with Fisher-Hayter least significant difference procedure). SMA, Simultaneous multiantibody; VEC, vascular endothelial cell; SMC, smooth muscle cell.

without enough supportive endothelial cells, can trigger the excessive proliferation of vascular VECs and an ensuing stenosis or block in some arteries.

No statistical difference was found among groups A, C, and $\mathrm{D}$ in pulmonary artery pressure, But in group $\mathrm{B}$, it is significantly lower than that in groups A, C, and D. The pressure in group $\mathrm{E}$ is more desirable than that in group B. According to histomorphologic analysis, to induce the establishment of pulmonary arterial collateral circulation and lower pulmonary arterial pressure, sequential transfection of Ad-hHGF and Ad-EGFP-hMCP-1 is recognized as being more effective than single or simultaneous transfection. The ratios of right ventricle weight index in groups E and B are significantly different, but the pulmonary artery pressure of these two groups shows no statistical difference. A possible explanation is that the hemodynamic alteration may occur after the structural change, which did not appear by the end of our experimental time frame, or that our hemodynamic examination is not precise enough to reveal these changes.

This study additionally suggests that groups D and E differ significantly in that the latter is more helpful for the development of blood vessels. Therefore, choosing the appropriate moment to transfect MCP-1 is critical. In group $\mathrm{D}$, the proliferation, chemotaxis, and coupling of endothelial cells synchronize those of the VECs, violating 


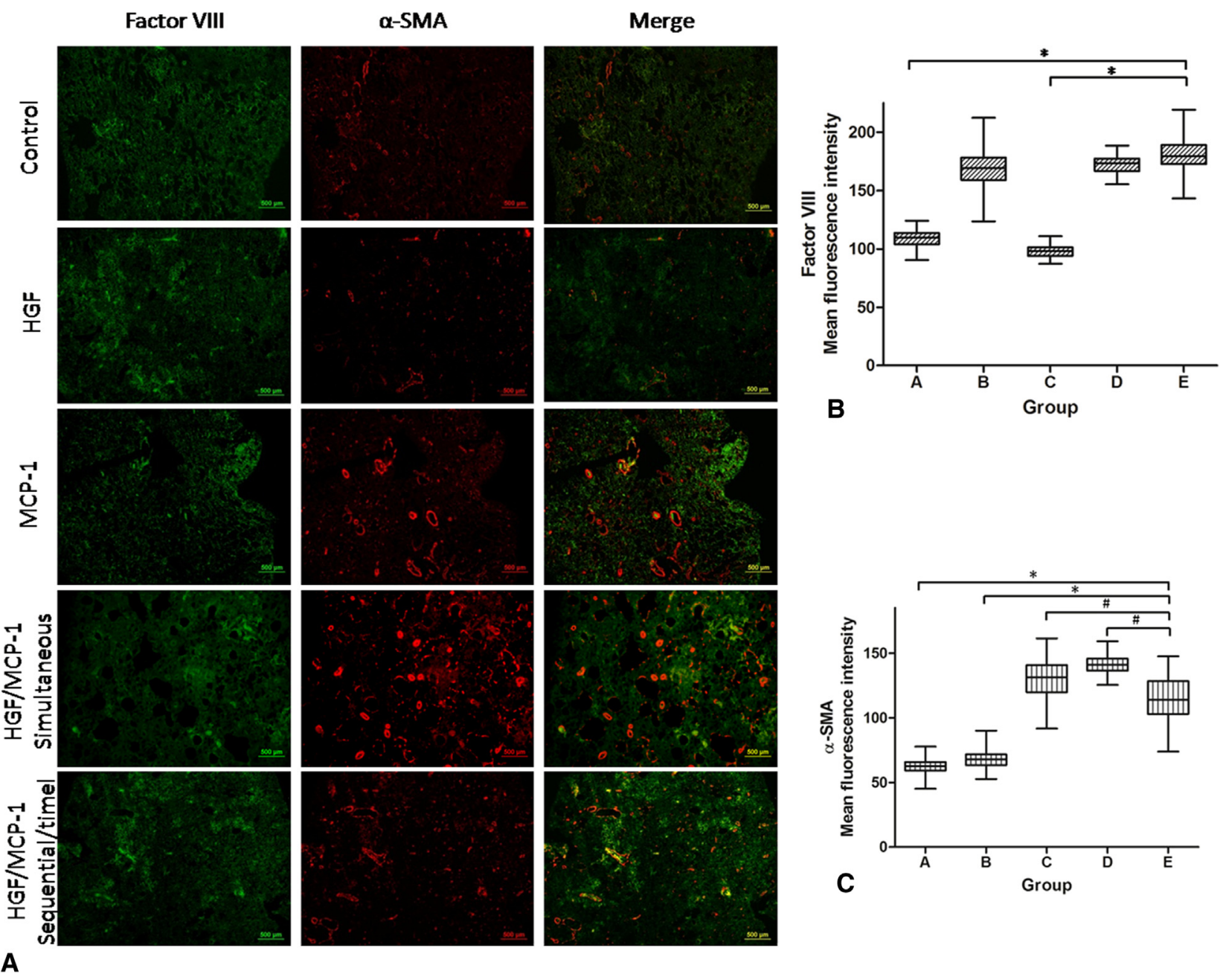

FIGURE 4. The microvascular density and the distribution of arterioles ( $\mathrm{n}=100$ slices per group). A, Factor VIII and $\alpha$-SMA immunofluorescence double staining were used to evaluate the microvascular density and the distribution of arterioles. B, Semiquantitative analysis of the factor VIII immunofluorescence examination. Groups B, D, and E had significantly higher intensity than groups A and C $(* P<.05)$. C, Semiquantitative analysis of the $\alpha$-SMA immunofluorescence examination. The mean fluorescence intensity of $\alpha$-SMA in group $\mathrm{E}$ was stronger than it was in groups $\mathrm{A}$ and $\mathrm{B}(* P<.05)$, but weaker than in groups $\mathrm{C}$ and $\mathrm{D}(\# P<.05)$. (ANOVA of factor VIII: $\mathrm{F}=1430.93, P<.01$; ANOVA of $\alpha$-SMA: $\mathrm{F}=1124.37, P<.01 ; *$ and $\#, P<.05,1$-way ANOVA with Fisher-Hayter least significant difference procedure). SMA, Simultaneous multiantibody; $H G F$, hepatocyte growth factor; $M C P-1$, monocyte chemotactic protein-1 gene.

the normal process of angiogenesis. With MCP-1, chemotaxis and adherence of macrophages will occur, and inflammatory factors will be secreted, all contributing to higher permeability of blood vessels. At the same time, MCP-1 induces VECs to multiply and migrate, which thickens the medial muscular layer of arteries. These muscle cells, after passing through the endothelium into the arterial lumens, continue to thrive there until arterial stenosis and even blocking occur.

In group $\mathrm{E}$, with a well-structured capillary evolving from endothelial cells via early transfection of HGF, MCP-1 is thereafter used to promote the proliferation and chemotaxis of VECs. Around the newborn vessels containing complete intimal structures, those VECs grow into the medial layer of arterioles, rather than migrate into the lumen. Additionally, in the later phase of angiogenesis, the original vessels without lumen or bloodstream will degenerate. The vessels without complete intimal structure and with higher permeability will be blocked by the proliferating and migrating VECs.

These phenomena are confirmed by our observations in this study. In group E, the number of endothelial cells is close to that in group B; its vascular density is higher than that in the control group and lower than that in group B; and its number of arterioles with unobstructed lumens and smooth muscle layers is significantly larger than that in any other groups. The sequential transfection of HGF and MCP-1 is a process similar to the physiology of newborn 
vessels. Later transfection of MCP-1 can help newborn vessels develop into arterioles that are essential to the construction of collateral circulation.

Therefore, during the processes of angiogenesis, the transfection of MCP-1, if started too early, will reduce the number of vessels with unobstructed lumens; if started too late, it will prolong illness and cause more animal deaths. The best time to use MCP-1 is, first, when formerly transfected HGF has already induced a large number of vessels equipped with complete endothelial membranes. Previous experiments on animals proved that angiogenesis can be observed clearly 1 week after the transfection of Ad-HGF; and after 2 weeks, capillary-like microvessels can be morphed. The second time is when specific antibodies, products of the body's immune response triggered by adenoviruses carrying target genes, have inactivated Ad-EGFP-MCP-1. So, in the sequential therapeutic system, virus vectors, to avoid being activated by the immune system, should not be used during the time in which antibodies within the plasma enjoy high valence values. Previous studies have provided convincing evidence that HGF can either facilitate pulmonary vessels to develop, or lower pulmonary arterial pressure.

As this study demonstrated, sequentially transfected HGF and MPC-1 could decrease pulmonary arterial pressure, and the newborn smooth muscle could prop up the blood vessels well. However, 1 limitation of this study is the lack of observation lasting $>6$ months; study on the effect of MPC-1 on the arterialization of newborn blood vessels needs to be conducted over a longer time period. Another limitation is that results from rabbit models may differ when human HGF and MPC are transfected. Additionally, the molecular mechanism of the effect of sequentially transfected HGF and MPC-1 on the arterialization of newborn blood vessels remains to be determined.

\section{CONCLUSIONS}

Hepatocyte growth factor and MCP-1 were expressed in rabbit lung after gene transfection via an airway approach. By increasing the pulmonary microvascular density and promoting microvascular arterializations, sequential and timely HGF/MCP-1 transfection ameliorates the PAH induced by shunt flow.

\section{Conflict of Interest Statement}

Authors have nothing to disclose with regard to commercial support.

The authors thank Dr Wu Zuze for the donation of recombinant adenovirus vector and for technologic guidance.

The authors thank Lang Zhuo (Department of Epidemiology, School of Public Health, Xuzhou Medical College) for statistical guidance.

\section{References}

1. Roos-Hesselink JW, Meijboom FJ, Spitaels SE, Van Domburg R, Van Rijen EH, Utens EM, et al. Outcome of patients after surgical closure of ventricular septal defect at young age: longitudinal follow-up of 22-34 years. Eur Heart J. 2004;25: 1057-62.

2. Chen G, He JG, Liu ZH, Gu Q, Ni XH, Zhao ZH, et al. Clinical features of adult patients with Eisenmenger syndrome associated with different types of congenital heart disease. Zhonghua Yi Xue Za Zhi. 2013;93:1546-9.

3. White AR, Ryoo S, Li D, Champion HC, Steppan J, Wang D, et al. Knockdown of Arginase I restores NO signaling in the vasculature of old rats. Hypertension. 2006; 47:245-51.

4. Thomas IC, Glassner-Kolmin C, Gomberg-Maitland M. Long-term effects of continuous prostacyclin therapy in adults with pulmonary hypertension associated with congenital heart disease. Int J Cardiol. 2013;168:4117-21.

5. Bui MT, Grollmus O, Ly M, Mandache A, Fadel E, Decante B, et al. Surgical palliation of primary pulmonary arterial hypertension by an unidirectional valved Potts anastomosis in an animal model. J Thorac Cardiovasc Surg. 2011;142: 1223-8.

6. Waddell TK, Bennett L, Kennedy R, Todd TR, Keshavjee SH. Heart-lung or lung transplantation for Eisenmenger syndrome. J Heart Lung Transplant. 2002;21: 731-7.

7. Adatia I, Kothari SS, Feinstein JA. Pulmonary hypertension associated with congenital heart disease: pulmonary vascular disease: the global perspective. Chest. 2010;137:52-61.

8. Galie N, Manes A, Palazzini M, Negro L, Marinelli A, Gambetti S, et al. Management of pulmonary arterial hypertension associated with congenital systemic-to-pulmonary shunts and Eisenmenger's syndrome. Drugs. 2008;68: 1049-66.

9. Cristofaro B, Stone OA, Caporali A, Dawbarn D, Ieronimakis N, Reyes M, et al. Neurotrophin-3 is a novel angiogenic factor capable of therapeutic neovascularization in a mouse model of limb ischemia. Arterioscler Thromb Vasc Biol. 2010;30:1143-50.

10. Oostendorp M, Douma K, Wagenaar A, Slenter JM, Hackeng TM, van Zandvoort MA, et al. Molecular magnetic resonance imaging of myocardial angiogenesis after acute myocardial infarction. Circulation. 2010; 121:775-83.

11. Van Belle E, Witzenbichler B, Chen D, Silver M, Chang L, Schwall R, et al. Potentiated angiogenic effect of scatter factor/hepatocyte growth factor via induction of vascular endothelial growth factor: the case for paracrine amplification of angiogenesis. Circulation. 1998;97:381-90.

12. Hosokawa Y, Hosokawa I, Ozaki K, Nakae H, Matsuo T. Increase of CCL20 expression by human gingival fibroblasts upon stimulation with cytokines and bacterial endotoxin. Clin Exp Immunol. 2005;142:285-91.

13. Kaga T, Kawano H, Sakaguchi M, Nakazawa T, Taniyama Y, Morishita R Hepatocyte growth factor stimulated angiogenesis without inflammation: differential actions between hepatocyte growth factor, vascular endothelial growth factor and basic fibroblast growth factor. Vascul Pharmacol. 2012;57:3-9.

14. Wang W, Liu K, Zhang F, Cao G, Zhang Y, Liu R, et al. Recombinant human hepatocyte growth factor transfection alleviates hyperkinetic pulmonary artery hypertension in rabbit models. J Thorac Cardiovasc Surg. 2013;146: 198-205.

15. Boomsma RA, Geenen DL. Mesenchymal stem cells secrete multiple cytokines that promote angiogenesis and have contrasting effects on chemotaxis and apoptosis. PLoS One. 2012; 7:e35685.

16. Nickerson MM, Burke CW, Meisner JK, Shuptrine CW, Song J, Price RJ. Capillary arterialization requires the bone-marrow-derived cell (BMC)-specific expression of chemokine (C-C motif) receptor-2, but BMCs do not transdifferentiate into microvascular smooth muscle. Angiogenesis. 2009;12: 355-63.

17. Wang W, Liu R, Cao G, Zhang F, Zhang Y, Zhang Z. A reliable rabbit model for hyperkinetic pulmonary hypertension. J Thorac Cardiovasc Surg. 2010;140:395-9.

18. Ha X, Yuan B, Li Y, Lao M, Wu Z. Gene therapy for pathological scar with hepatocyte growth factor mediated by recombinant adenovirus vector. Sci China C Life Sci. 2003;46:320-7.

19. Liu C, Yan Z, Fang C, Cao G, Wang B, Li S, et al. Establishment and comparison of two reliable hyperkinetic pulmonary hypertension models in rabbits. J Thorac Cardiovasc Surg. 2014;148:2353-9.

20. Louzier V, Eddahibi S, Raffestin B, Déprez I, Adam M, Levame M, et al. Adenovirus-mediated atrial natriuretic protein expression in the lung protects rats from hypoxia-induced pulmonary hypertension. Hum Gene Ther. 2001;12: $503-13$. 
21. Liu K, Liu R, Cao G, Sun H, Wang X, Wu S. Adipose-derived stromal cell autologous transplantation ameliorates pulmonary arterial hypertension induced by shunt flow in rat models. Stem Cells Dev. 2011;20:1001-10.

22. Tsai CS, Lin FY, Chen YH, Yang TL, Wang HJ, Huang GS, et al. Cilostazol attenuates MCP-1 and MMP-9 expression in vivo in LPS-administrated balloon-injured rabbit aorta and in vitro in LPS-treated monocytic THP-1 cells. J Cell Biochem. 2008;103:54-66.

23. Ishimaru K, Miyagawa S, Fukushima S, Saito A, Sakai Y, Ueno T, et al. Synthetic prostacyclin agonist, ONO1301, enhances endogenous myocardial repair in a hamster model of dilated cardiomyopathy: a promising regenerative therapy for the failing heart. J Thorac Cardiovasc Surg. 2013; 146:1516-25.

24. Shimamura M, Sato N, Yoshimura S, Kaneda Y, Morishita R. HVJ-based non-viral gene transfer method: successful gene therapy using HGF and VEGF genes in experimental ischemia. Front Biosci. 2006; $11: 753-9$

25. Ono M, Sawa Y, Matsumoto K, Nakamura T, Kaneda Y, Matsuda H. In vivo gene transfection with hepatocyte growth factor via the pulmonary artery induces angiogenesis in the rat lung. Circulation. 2002;106:I264-9.

26. Kato N, Nemoto K, Nakanishi K, Morishita R, Kaneda Y, Uenoyama M, et al. Nonviral gene transfer of human hepatocyte growth factor improves streptozotocin-induced diabetic neuropathy in rats. Diabetes. 2005;54 846-54.

27. Ono M, Sawa Y, Fukushima N, Suhara H, Nakamura T, Yokoyama C, et al. Gene transfer of hepatocyte growth factor with prostacyclin synthase in severe pulmonary hypertension of rats. Eur J Cardiothorac Surg. 2004;26:1092-7.

28. Wang W, Wu SM, Zhang ZM, Zhang YQ. In vivo transfection of hepatocyte growth factor gene induces pulmonary angiogenesis in rabbits with hyperkinetic pulmonary hypertension. Zhonghua Xin Xue Guan Bing Za Zhi. 2008;36:1110-4.

29. Rangasamy S, McGuire PG, Franco Nitta C, Monickaraj F, Oruganti SR, Das A Chemokine mediated monocyte trafficking into the retina: role of inflammation in alteration of the blood-retinal barrier in diabetic retinopathy. PLoS One. 2014; 9:e108508.

30. Spinetti G, Wang M, Monticone R, Zhang J, Zhao D, Lakatta EG. Rat aortic MCP-1 and its receptor CCR2 increase with age and alter vascular smooth muscle cell function. Arterioscler Thromb Vasc Biol. 2004;24:1397-402.

Key Words: hepatocyte growth factor (HGF), monocyte chemotactic protein-1 (hMCP-1), pulmonary artery hypertension $(\mathrm{PAH})$, angiogenesis, microvascular arterialization

\section{EDITORIAL COMMENTARY}

\section{A new outlet for pulmonary arterial hypertension}

\section{Chadrick E. Denlinger, MD}

See related article on pages 634-43.

Pulmonary arterial hypertension (PAH) is a rare condition, with an incidence of 1 to 2 cases per million people in the United States. The risk for PAH is 2 to 4 times greater among women than men, and PAH is also associated with systemic sclerosis, human immunodeficiency virus infection, portal hypertension, and chronic cardiac shunts causing pulmonary overcirculation. At least $40 \%$ of cases PAH are idiopathic, however, with no identified related syndrome. During the past 2 decades, PAH has become more manageable, with numerous treatment options becoming available. Since the 1990s, calcium channel blockers have been recommended by the American College of Chest

From the Division of Cardiothoracic Surgery, Medical University of South Carolina, Charleston, SC.

Disclosures: Author has nothing to disclose with regard to commercial support.

Received for publication May 25, 2015; accepted for publication May 26, 2015; available ahead of print June 24, 2015.

Address for reprints: Chadrick E. Denlinger, MD, 114 Doughty St, Suite 201,

Charleston, SC 29425 (E-mail: denlinge@musc.edu).

J Thorac Cardiovasc Surg 2015;150:643-4

$0022-5223 / \$ 36.00$

Copyright (c) 2015 by The American Association for Thoracic Surgery

http://dx.doi.org/10.1016/j.jtcvs.2015.05.059
Physicians for patients with $\mathrm{PAH}$ who have a favorable response to the administration of vasodilators. ${ }^{1}$ Unfortunately, only $10 \%$ of the population have an acceptable response to vasodilators and thus

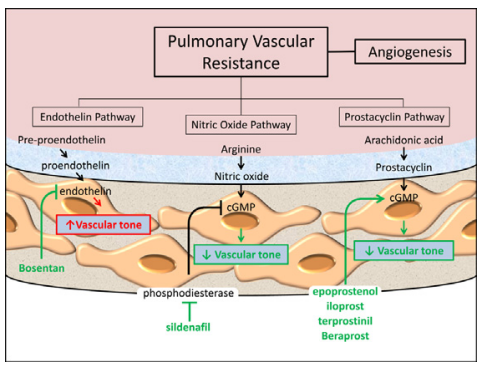
are eligible for this treatment. A more thorough understanding of the pathophysiology of this disease has ushered in a variety of additional treatment options.

Three predominant pathways have emerged with pathologic roles in the development of PAH. Deficiencies of naturally occurring vasodilatory prostacyclins and nitric oxide or an abundance of the vasoconstrictive endothelins may each contribute to $\mathrm{PAH}$. A relative deficiency of prostacyclins, which have vasodilatory and antiplatelet effects, has been recognized as a contributing factor in the pathogenesis of PAH. For this reason, several prostacyclin analogs have emerged as treatment options for patients with advanced PAH. Prospective, randomized studies have demonstrated a reduction in the pulmonary arterial pressure in response to prostacyclins. ${ }^{2}$ Patients also demonstrate improvements in exercise tolerance and quality of life when treated. 


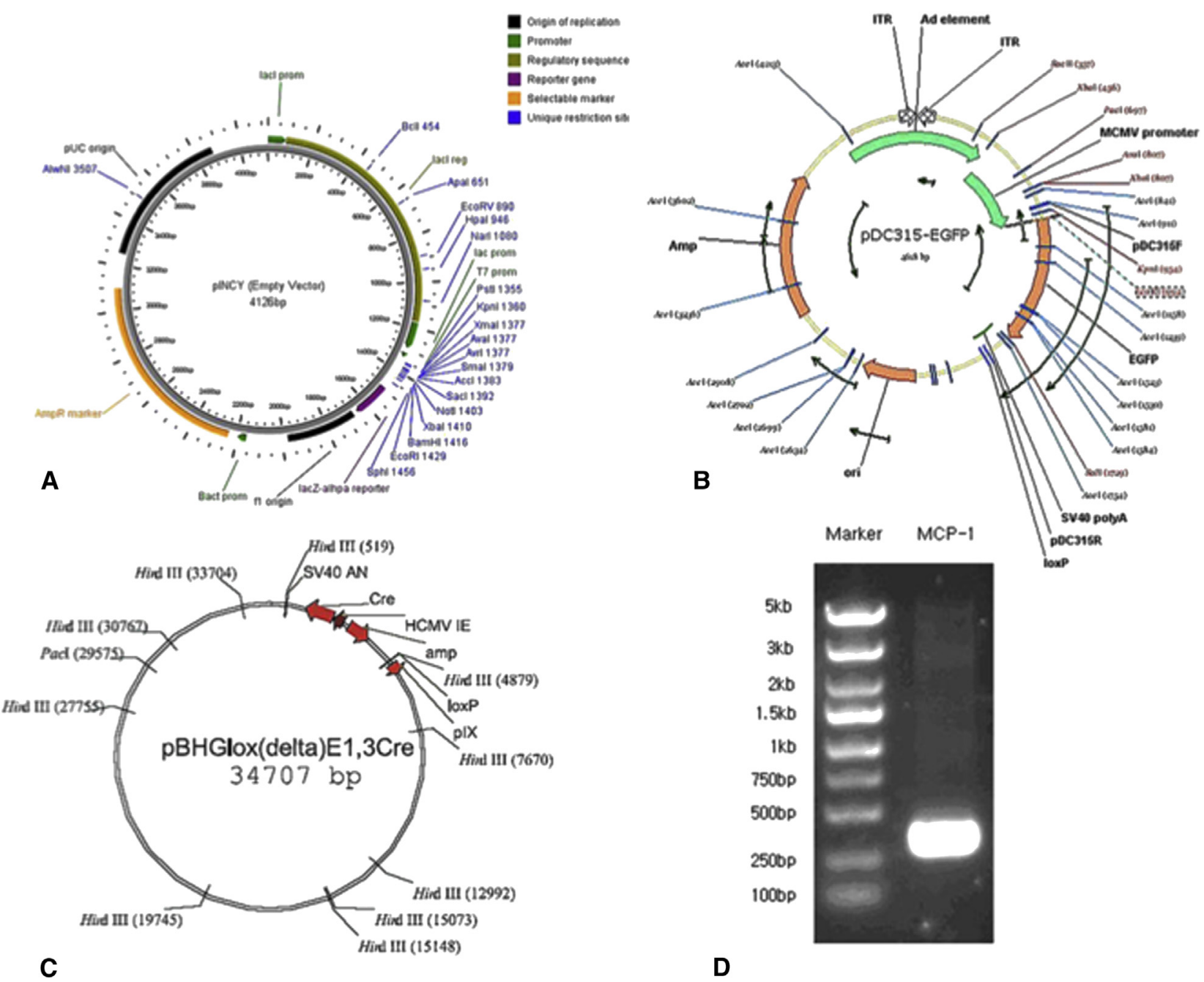

FIGURE E1. Structures of 3 plasmids of recombinant adeno-associated viral vectors. A, pINCY. B, pBGH lox $\Delta \mathrm{e} 1,3$ cre. C, pDC315-EGFP-hMCP-1. $\mathrm{D}$, Polymerase chain reaction product of hMCP-1. AMP, Adenosine monophosphate; ITR, inverted terminal repeats; $M C M V$, murine cytomegalovirus; $E G F P$, enhanced green fluorescent protein; $M C P-1$, monocyte chemotactic protein-1; HCMV, human cytomegalovirus.

TABLE E1. Experimental group classification

\begin{tabular}{|c|c|c|c|c|}
\hline Group A $(n=15)$ & Group B $(\mathbf{n}=15)$ & Group C $(n=15)$ & Group D $(n=15)$ & Group E $(n=15)$ \\
\hline Control & $\begin{array}{l}\text { hHGF/Ad-null sequential, } \\
\text { timely }\end{array}$ & $\begin{array}{l}\text { hMCP-1/Ad-null sequential, } \\
\text { timely }\end{array}$ & hHGF/hMCP-1 simultaneous & $\begin{array}{l}\text { hHGF/hMCP-1 sequential, } \\
\text { timely }\end{array}$ \\
\hline
\end{tabular}

Sequential, timely treatment was transfection of the prior and posterior Ad-target gene or Ad-null, with an interval of 1 week. $h H G F$, Human hepatocyte growth factor; $h M C P-1$, human monocyte chemotactic protein-1 gene; Ad-null, adenovirus containing no gene in the expression cassette. 
TABLE E2. Analysis of variance for numbers of VECs and SMCs

\begin{tabular}{|c|c|c|c|c|c|}
\hline Variable & Group A & Group B & Group C & Group D & Group E \\
\hline $\mathrm{n}$ & 20 & 20 & 20 & 20 & 20 \\
\hline VECs & $9.04 \pm 1.69^{*}$ & $19.25 \pm 1.96^{*}$ & $9.24 \pm 1.03^{*}$ & $15.44 \pm 1.47 *$ & $17.33 \pm 1.99$ \\
\hline SMCs & $4.61 \pm 0.3^{*}$ & $2.86 \pm 0.53^{*}$ & $7.28 \pm 0.49 *$ & $7.52 \pm 0.59^{*}$ & $5.46 \pm 0.63$ \\
\hline
\end{tabular}

TABLE E3. Results of ANOVA of immunofluorescence density of factor VIII and $\alpha$-SMA

\begin{tabular}{lccccc}
\hline Variable & Group A & Group B & Group C & Group D & Group E \\
\hline $\mathrm{n}$ & 100 & 100 & 100 & 100 & 100 \\
Factor VIII & $109.31 \pm 6.69^{*}$ & $169.29 \pm 15.03^{*}$ & $98.02 \pm 5.1^{*}$ & $172.69 \pm 7.51^{*}$ & $180.29 \pm 13.32$ \\
$\alpha$-SMA & $62.29 \pm 6.11^{*}$ & $67.72 \pm 6.4^{*}$ & $131.13 \pm 13.6^{*}$ & $141.28 \pm 6.69^{*}$ & $115.04 \pm 16.77$ \\
\hline Factor VIII ANOVA: $\mathrm{F}=1430.93, P<.01 ;$ ANOVA of $\alpha$-SMA: $\mathrm{F}=1124.37, P<.01 . S M A$, Simultaneous multiantibody. $* P<.05$, compared with group E, determined by the \\
Fisher-Hayter least significant difference procedure.
\end{tabular}

